\title{
Sur quelques marques de subjectivité dans le journalisme d'information politique de 1945 à 2015 au Québec
}

On some subjectivity marks in political information journalism from 1945 to 2015 in Quebec

Algunas marcas de subjetividad en el periodismo de información política de 1945 a 2015 en Quebec

Louise Chaput

\section{OpenEdition}

Journals

Édition électronique

URL : https://journals.openedition.org/mots/24586

DOI : $10.4000 /$ mots. 24586

ISSN : 1960-6001

Éditeur

ENS Éditions

Édition imprimée

Date de publication : 5 mars 2019

Pagination : 151-168

ISBN : 979-10-362-0130-1

ISSN : 0243-6450

\section{Référence électronique}

Louise Chaput, «Sur quelques marques de subjectivité dans le journalisme d'information politique de 1945 à 2015 au Québec », Mots. Les langages du politique [En ligne], 119 | 2019, mis en ligne le 01 janvier 2022, consulté le 22 avril 2022. URL : http://journals.openedition.org/mots/24586 ; DOI : https://doi.org/10.4000/mots.24586 


\section{Sur quelques marques de subjectivité dans le journalisme d'information politique de 1945 à 2015 au Québec}

Se fondant sur un corpus de nouvelles tirées de deux organes de presse québécois, La Presse et Le Devoir (1945-1995), Jean Charron s'est consacré à l'étude de quatre indicateurs de la subjectivité énonciative dans la nouvelle journalistique : attributions d'états psychologiques, connecteurs logiques, modalités subjectives et énoncés prospectifs. Ses travaux lui ont permis de constater « un net déplacement, à partir des années 1960 et 1970, de la posture énonciative des journalistes de l'objectivité vers la subjectivité » (Charron, 2006a, résumé p.131). Il décrit ainsi ce concept de subjectivité :

Le locuteur tend à la subjectivité dès qu'il se met en scène lui-même en tant que sujet dans son énonciation, lorsqu'il engage plus ou moins manifestement son identité en tant que sujet du discours, lorsqu'il rend perceptible, par ses choix langagiers, le fait que l'énoncé est lié au locuteur. (Charron, Jacob, 1999, p. 10)

Ainsi, le journalisme d'information aurait subi des transformations : au souci de l'objectivité aurait succédé une pratique journalistique plus teintée de subjectivité et plus axée sur l'interprétation des événements, leur analyse et la spéculation quant à leurs causes et à leurs conséquences (Brin, Charron, Bonville éd., 2004). Cette pratique mobilise une rhétorique d'expertise critique qui se caractérise par une mise en perspective des événements politiques par le biais de données, d'analyses et de jugements de la part du journaliste.

Au style expressif du journalisme d'opinion correspond en contrepartie l'écriture instrumentale et documentaire de l'expertise critique. Les jugements accompagnés de données factuelles apparaissent dépouillés du caractère saillant des propos d'opinion. Le journalisme d'opinion cherche à convaincre, la rhétorique de l'expertise critique s'efforce de persuader. (Padioleau, 1976, p. 277)

Nous nous efforçons ici de prolonger l'étude de J. Charron en travaillant sur le même corpus de presse (voir ci-dessous), sur une période plus longue 
(jusqu'en 2015), et en examinant des indicateurs plus fins à la lumière de deux concepts empruntés à Dominique Maingueneau.

Le premier, la «tension», a trait aux formes linguistiques auxquelles un énonciateur peut recourir pour exercer une certaine influence sur le lecteur ou établir un contact avec celui-ci. Selon D. Maingueneau, «le concept de tension regarde la relation qui s'établit entre le locuteur et l'allocutaire, le texte étant considéré comme médiateur d'un désir du locuteur, tentative de saisie de l'allocutaire» (Maingueneau, 1976, p.120).

Précisons que, dans le cas de la nouvelle, en tant que genre institué où l'énonciateur est limité par son rôle d'informateur, le journaliste ne dispose pas de la même latitude que l'auteur d'articles d'opinion ou de commentaires en ce qui regarde les moyens qu'il peut employer pour interpeller son lecteur et, par le fait même, pour laisser paraître un engagement par rapport à son énoncé.

Le second concept est celui de la «distance», qui renvoie à d'autres indicateurs de subjectivité au sujet desquels D. Maingueneau apporte la précision suivante :

Si cette distance tend vers zéro, le sujet prend totalement en charge l'énoncé [...]. Malheureusement, le fait fondamental est qu'il n'existe pas de moyens privilégiés, de classes de mots particuliers pour signifier cette distance : telle structure repérée se révèle l'instant suivant avoir une autre signification. (Maingueneau, 1976, p.119)

La prise en charge dont il est question ici peut prendre la forme, notamment, d'une prise de position, d'une opinion (plus ou moins explicite) du journaliste destinée à susciter l'adhésion du lecteur ou à soulever un questionnement chez ce dernier. Ce positionnement dans l'énonciation participe à la « présentation de soi » à travers le discours pour produire un effet sur le public (Amossy, 2010).

Nous travaillerons donc sur les manifestations de quatre indicateurs linguistiques de la «tension » et de la « distance » observables au fil des ans dans les textes produits par le journalisme d'information. Notre analyse se limitera au plan discursif, mais nous proposerons tout de même dans la dernière section des pistes explicatives d'ordre historique et sociologique.

\section{Corpus et méthodologie}

Cette recherche s'inscrit dans une perspective diachronique : comme celle de J. Charron, elle s'appuie sur un corpus d'articles d'information de politique locale, provinciale ou nationale (à l'exclusion des textes provenant d'agences) parus dans les quotidiens La Presse et Le Devoir de 1945 à 2015², à raison d'une année par décennie (1945, 1955...), et de 70 à 80 articles par année. Il

1. Corpus rassemblé par le Groupe de recherche sur les mutations du journalisme (GRMJ) du Département d'information et de communication de l’Université Laval. 
s'agit d'un échantillonnage établi à partir du calendrier perpétuel en ligne pour chaque année retenue (http://eldiade.com/fr/) et d'un tableau créé avec des nombres aléatoires correspondant à une date, une semaine et un mois, c'est-àdire la méthode des «semaines construites » qui retient une édition au hasard pour chaque jour de la semaine. Ce corpus comprend 580 articles de La Presse et 624 articles du Devoir. Ces deux quotidiens n'ont jamais eu la même ligne éditoriale : celle de La Presse, de tendance fédéraliste, est demeurée plutôt constante, alors que celle du Devoir a évolué avec les années vers une position plus nationaliste. De plus, le lectorat ciblé par les deux quotidiens n'est pas le même : La Presse est connue pour son ton assez populiste, tandis que Le Devoir est réputé pour son approche plus intellectuelle.

Notre démarche consiste à relever les fréquences d'indicateurs de «tension » - éléments linguistiques visant à établir une relation entre l'auteur et le lecteur et celles des marques de « distance» - éléments indiquant la prise en charge de l'énoncé par le rédacteur, ce qui accroît le degré d'engagement de celui-ci dans son texte. Nous appellerons aussi ces indicateurs «marques de rapprochement» (tension accrue) et «marques d'engagement» (distance réduite), expressions plus concrètes que nous considérons comme moins ambiguës dans certains contextes. Les fréquences de ces marques détermineront la variation de leur ampleur d'une décennie à l'autre afin de vérifier si le journalisme d'information connaît une évolution. Ces résultats pourraient corroborer ceux des études de J. Charron, qui ont affiché une nette évolution du recours à quatre autres indicateurs linguistiques de subjectivité dans les années 1960 et 1970 dans le journalisme d’information politique au Québec (Charron, 2006a).

\section{Variables linguistiques de rapprochement et d'engagement}

Parmi les multiples façons de traiter les marques de rapprochement entre le journaliste et son lectorat (tension accrue) et d'engagement du journaliste, c'est-à-dire la prise en charge de son énoncé (distance réduite), notions décrites dans l'introduction, nous avons retenu quatre indicateurs linguistiques dont la présence dans la presse écrite pouvait se révéler significative : les points de suspension; les tirets et les parenthèses; les guillemets de distanciation. Ces ponctèmes constituent des marques linguistiques de procédés discursifs qui traduisent à la fois, et à des degrés divers, un engagement du locuteur et un rapprochement de celui-ci avec l'allocutaire :

- les points de suspension : le rédacteur établit une certaine connivence avec son lecteur en le supposant assez compétent pour compléter l'énoncé;

- la parenthèse de commentaire : le rédacteur prend position et tente de persuader le lecteur ou d'influer sur sa pensée;

- les tirets de mise en relief : le rédacteur met en relief un commentaire ou 
un élément d'information qu'il juge important pour attirer l'attention du lecteur;

- les guillemets de mise à distance : le rédacteur signale une expression ou un mot dont il veut se distancier et, par conséquent, amène le lecteur à y porter attention².

Nous formulons l'hypothèse que, dans les nouvelles des deux quotidiens à l'étude, soit La Presse et Le Devoir, le taux d'occurrence de ces indicateurs linguistiques a augmenté, confirmant la thèse selon laquelle le journalisme d'information fait une place de plus en plus importante à la subjectivité des journalistes.

\section{La suspension}

Les trois points font référence au non-dit. Ils peuvent servir à clore une énumération incomplète (énoncé tronqué), prenant alors la même valeur qu'etc. Dans ces cas ont été calculés, par dix mille mots, des taux d'occurrence très faibles dans La Presse (0,51 en 1945, 0,83 en 1965, 0,37 en 1975 et 0,23 en 1995), et encore moins importants dans Le Devoir (0,17 en 1955, 0,3 en 1975 et 0,3 en 1985).

Dans les nouvelles, les points de suspension signalent aussi et surtout le choix du journaliste de ne pas terminer l'idée qu'il a commencé à exprimer ou sa réticence à dévoiler toute sa pensée : le lecteur devient ainsi responsable de l'interprétation du non-exprimé, du sous-entendu. Ils peuvent également servir à insérer une pause prenant la valeur d'une phrase incidente s'adressant à l'interlocuteur («devinez», «je vous le donne en mille», «est-ce possible?», «nul besoin d'en dire plus»). Ces emplois sous-entendent donc un commentaire ou une remarque du rédacteur sur l'information fournie. En voici un exemple :

Ils [des engagements du gouvernement] seront réalisés généralement sur des périodes variant entre trois et cinq ans. L'exception de taille : les huit milliards que coûtera la baisse des loyers des aéroports. On parle ici d'une facture étalée sur... 50 ans. Mais, au moment de l'annonce, le ministre des Transports, Jean Lapierre, était bien heureux de souligner ce chiffre ronflant. (Manon Cornellier, Le Devoir, 28 mai 2005)

Le journaliste juge donc son lecteur assez informé ou compétent pour comprendre l'information véhiculée par les points de suspension : ceux-ci peuvent exprimer de nombreux effets de sens et leur aptitude à se substituer à un ou plusieurs composants syntaxiques se révèle d'une grande commodité pour les rédacteurs. Le caractère imprécis des points de suspension permet ainsi au journaliste de suggérer des pistes de réflexion sans risquer d'être accusé de manquer d'objectivité.

2. Procédé ancien apparu avec l'invention de l'interview journalistique (Schudson, 1996, p. 72-93). 
On retrouve les points de suspension tant à l'intérieur (53 occurrences) qu'à la fin de la phrase (54 occurrences). Bien qu'ils soient peu nombreux, leur présence est significative. Figurant au milieu de l'énoncé, ils

peuvent créer un effet d'attente - de suspense en quelque sorte - destiné à préparer le lecteur à intégrer une nouvelle d'emblée ressentie comme imprévisible [...]. Pure théâtralisation d'une interaction qui se donne à voir ostensiblement, et qui légitime le report du travail inférentiel. (Dalhet, 2003, p.105; les caractères gras sont de l'auteur)

En réalité, c’est au lecteur de concevoir des interprétations qui ne sont pas forcément évidentes, car l'inférence dépend, d'une part, des connaissances de celui-ci et, d'autre part, de l'information fournie par l'énonciation. Le rédacteur sollicite donc la participation du lecteur (tension accrue). À la fin d'un énoncé, les points suspensifs pourraient commuter avec le point, mais leur usage implique que tout n'est pas dit. L'ellipse manifestée par les points de suspension peut insinuer un fait que le journaliste trouve louche mais sur lequel il ne peut apporter de preuves :

Robert Gabriel [responsable de la sécurité publique] est le fils de Léonard Gabriel, le propriétaire des serres où la marijuana aurait été semée avant d'être mise en terre. Interrogé sur ce lien de parenté, il a dit ne pas vouloir mêler sa famille avec ses fonctions officielles à Kanesatake... (François Berger, La Presse, 26 juillet 1995)

Dans l'énoncé suivant, les points de suspension renforcent l'ironie qui caractérise le propos:

Les réseaux français et anglais de Radio-Canada assureront la diffusion complète de ces deux soirées sous le titre, au réseau français, de "L'élection du chef du Parti québécois». Au réseau anglais de la télévision d’État, on a dû prendre la décision pénible de repousser à minuit, le dimanche, la première de la nouvelle saison de la série "Dallas» qui connaît le succès populaire que l'on sait... (Mario Roy, La Presse, 12 septembre 1985)

Bien que la télévision d’État soit obligée, en vertu de son mandat, de couvrir la course à la présidence d'un parti souverainiste, le journaliste, en utilisant les points de suspension, laisse entendre que ce sujet intéresse beaucoup moins les dirigeants du réseau anglais que les cotes d'écoute du feuilleton Dallas, sous-entendant que le Canada anglais porte peu d'intérêt à la question de la souveraineté du Québec. Le sous-entendu relève plutôt du commentaire et pourrait être omis sans changer le contenu informatif qui le précède.

Les points de suspension peuvent aussi traduire un doute :

Les conservateurs ont tourné la page sur l'ère de Stephen Harper en choisissant d'élire Rona Ambrose pour diriger leur parti dans l'intérim. Une femme, qui a promis un changement de ton... mais qui vient de l'Alberta et qui a pris trois questions des médias avant de tourner les talons. (Marie Vastel, Le Devoir, 6 novembre 2015) 
Le lecteur doit connaître la scène politique nationale pour bien comprendre que, malgré la promesse de changement de ton, la chef par intérim du Parti conservateur se montre tout aussi réticente que l'ancien Premier ministre à répondre aux questions des journalistes et à justifier les positions de son parti. La journaliste met en relief cette contradiction en insérant les points de suspension avant le marqueur d'opposition mais.

Malgré le caractère polysémique des points de suspension, les journalistes n'en font pas un usage très marqué, comme l'indique la figure suivante.

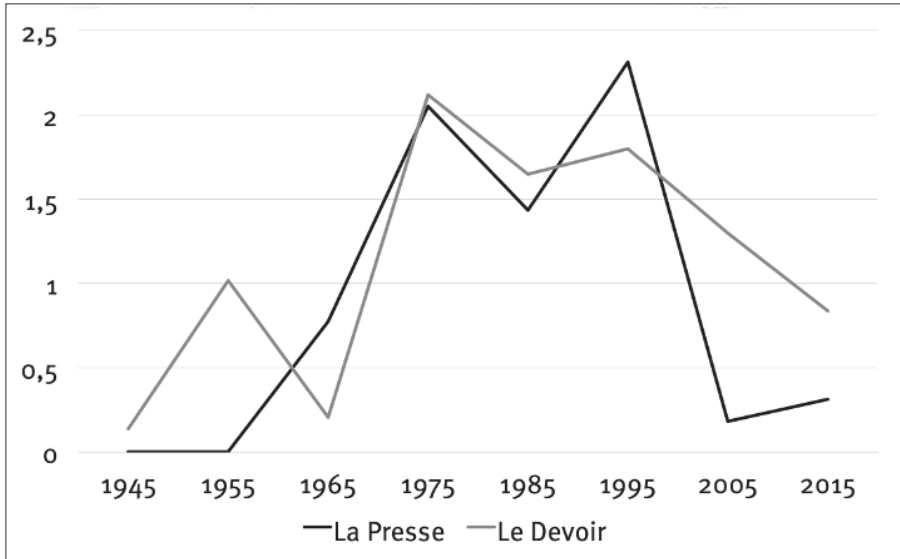

Figure 1. Taux d'occurrence par 10000 mots des points de suspension de 1945 à 2015

Le tableau montre une augmentation un peu plus prononcée des points de suspension à partir de 1975 dans La Presse, suivie d'une baisse en 1985 puis d'une remontée en 1995, mais les taux baissent considérablement dans les deux dernières décennies33. Quant au Devoir, le taux d'occurrence le plus élevé apparaît en 1975 et l'on constate une baisse, comme dans La Presse, en 2005 et en 2015, mais tout de même moins marquée. On peut conclure que les deux quotidiens, malgré leurs orientations différentes, présentent plus ou moins le même schéma d’évolution.

\section{La mise en relief par les tirets et le commentaire entre parenthèses}

Nous traitons dans la même section les tirets et les parenthèses, car ces signes de ponctuation ont des emplois apparentés : ils marquent une rupture en détachant ou en isolant des éléments. Ces segments détachés se trouvent alors à un autre niveau énonciatif, c'est-à-dire que le rédacteur s'adresse à son lecteur en interrompant la linéarité de son discours par l'insertion d'éléments d'infor-

3. Ce tableau ne comprend pas les occurrences des énoncés tronqués. 
mation ou d'un commentaire, non essentiels, mais que l'énonciateur juge utile d'ajouter afin d'aider le lecteur à mieux comprendre les faits ou la situation. Selon Sabine Pétillon-Boucheron, le décrochage est une «opération qui peut s’interpréter comme un “j’ajoute par ailleurs” [...], une opération énonciative d'ajout» (Pétillon-Boucheron, 2002, p.138). Guillaume François, quant à lui, rappelle que ces signes marquent un décrochage et un «dédoublement de la voix de l'énonciateur» (François, 2011, p. 3). Le dédoublement de la voix correspond à un certain positionnement ou à une réaction du journaliste par rapport aux informations qu'il a précédemment fournies, c'est-à-dire à un retour sur ce qui a été dit pour éclairer le lecteur avant de continuer ou de terminer son énoncé initial. Mais les tirets et les parenthèses sont-ils équivalents en ce qui a trait à leur force illocutoire et à leur importance énonciative? Nous traiterons d'abord des emplois des tirets pour ensuite les comparer avec ceux des parenthèses.

Pour Danielle Forget, le recours au tiret marque un rapprochement ou une tension accrue :

[Son insertion] souligne le souci du locuteur de pourvoir le destinataire de toute l'information nécessaire au décodage du message quand ce n'est pas une action directe qu'il entend exercer sur les conclusions visées ou les prémisses de son interprétation. Le renvoi au contenu du message à construire ou à l'interprétation en cours peut être plus ou moins explicite, mais il suppose et convoque la participation active du destinataire-interprétant. (Forget, 2000, p.19-20)

Le tiret double (placé à l'intérieur d'une phrase) ou simple (à la fin) marque une pause en mettant en relief des éléments détachés de diverses natures dans la phrase. Dans notre corpus de nouvelles, les éléments entre tirets ne forment jamais de phrase complète et sont dans la plupart des cas syntaxiquement dépendants de la phrase qui les accueille : il est rare que des séquences entre tirets produisent une rupture de linéarité. Les tirets servent à isoler des segments exprimant des précisions, des preuves, des explications, des exemples, des causes, des comparaisons, des commentaires, etc. Les informations, supplémentaires ou complémentaires, fournies dans ces segments sont suppressibles, leur retrait n'altérant pas la grammaticalité de la phrase qui les accueille ni le sens de l'énoncé principal.

L'exemple suivant illustre bien l'insistance du journaliste sur le caractère catégorique d'une décision prise par le gouvernement fédéral concernant une manifestation culturelle à Montréal.

Ottawa vient donc de refuser - et carrément - le projet de timbres-postes [sic] que lui soumettaient, sourire aux lèvres, les conseillers artistiques d’Expo 67. (Denis Masse, La Presse, 19 août 1965)

Le journaliste aurait pu simplement placer après le verbe refuser l'adverbe modalisateur carrément, mais en détachant ce dernier et en le mettant en relief 
avec la conjonction et, il mobilise plus efficacement l'attention du lecteur et révèle explicitement l'évaluation personnelle qu'il fait de ce refus.

Dans certains cas, la critique formulée peut être plus directe, le journaliste se positionnant en tant qu'évaluateur de l'information qu'il livre à ses lecteurs et, dans une certaine mesure, incitant les lecteurs à partager son opinion. Il est question dans l'exemple qui suit du discours de clôture de session du lieutenant-gouverneur, jugé par le journaliste tout aussi ennuyeux ("motto seriné ») que ceux quil l'ont précédé.

Et, évidemment, tout y a passé : lois améliorant le régime de vie des agriculteurs,

- vieux motto seriné depuis des temps immémoriaux - loi de la fonction publique,

lois sociales, lois des mines, etc. (Anonyme, La Presse, 7 août 1965)

L'utilisation des tirets, que les virgules pourraient remplacer dans la majorité des cas, ne se limite pas simplement à délimiter des segments présentant un complément d'information, mais peut également encadrer des commentaires visant à faire réfléchir le lecteur, comme l'illustre l'exemple suivant.

Il restera à la CSN pendant quelque temps - ce quelque temps pourrait être quelques semaines ou même quelques mois - et ensuite il sera «en disponibilité». (Evelyn Gagnon, Le Devoir, 8 avril 1965)

Dans l'exemple précédent, le commentaire entre tirets est pris en charge par la journaliste, qui souligne implicitement et avec une certaine ironie la rumeur voulant que $M$. Marchand, qui venait d'annoncer sa démission à titre de président de la CSN et était demeuré plutôt vague quant à son avenir (" ensuite il sera en disponibilité»), se lancerait en politique, ce qui se confirma quelques mois plus tard («ce quelque temps»).

La figure ci-dessous présente l'évolution du taux d'occurrence des tirets, calculé à partir des fréquences par dix mille mots, marquant la subjectivité au cours des décennies.

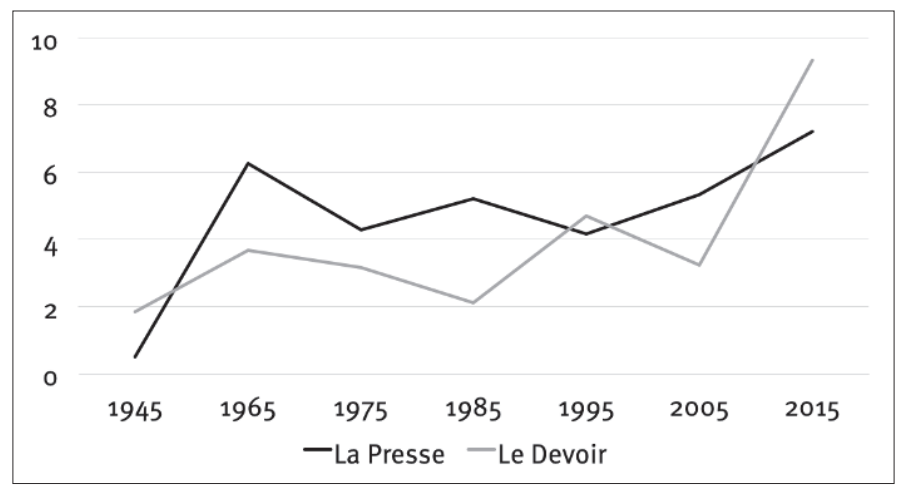

Figure 2. Taux d'occurrence par 10000 mots des tirets de 1945 à 2015 
Le graphique indique, pour les deux quotidiens, un accroissement assez important de l'usage des tirets en 1965, suivi d'une légère diminution puis d'une remontée, pour atteindre les taux d'occurrence maximaux en 2015. L'utilisation de ce ponctème pour marquer la subjectivité a donc subi une évolution au cours des décennies qui est attribuable à l'ensemble des journalistes.

En ce qui a trait aux parenthèses, à l'instar des tirets, elles permettent au rédacteur d'apporter des suppléments ou compléments d’information, ou encore de commenter la nouvelle. Toutefois, selon Robert De Beaugrande, elles se distinguent des tirets par leur caractère plus secondaire, de moindre importance (De Beaugrande, 1984, p. 204). Isabelle Serça, quant à elle, avance que "les parenthèses signaleraient un changement d'instance, avec une rupture énonciative franche » (Serça, 2010, p.101). Le changement d'instance rappelle le dédoublement de la voix par l'insertion d'un segment entre tirets : la voix qui informe et l'autre qui interrompt le discours pour faire un retour sur l'information donnée en apportant des précisions ou commentaires. G. François, pour sa part, se livrant à une fine analyse des divers usages de la parenthèse, observe que celle-ci peut se substituer au tiret, mais que l'inverse n'est pas toujours possible (François, 2011).

Dans notre corpus, on retrouve tant entre tirets qu'entre parenthèses tantôt des précisions (titres, fonctions, etc.), tantôt des commentaires du journaliste. Celui-ci peut se servir de l'un ou l'autre de ces signes pour rappeler des faits: «- on l'a déjà signalé - »; " (Rappelons que...) ». Cependant, les indications sur la réaction non verbale des gens et les renvois à d'autres sections du journal ou à d'autres textes sont présentés uniquement entre parenthèses : "éclats de rires dans la salle)»; "(voir à ce sujet nos informations à la page 16)». De telles informations ou indications, qui ne font pas réellement partie du message en soi, permettent au lecteur de mieux comprendre le contexte. Un autre emploi réservé exclusivement aux parenthèses est l'insertion d'une précision ou d'un commentaire à l'intérieur d'un discours direct rapporté, insertion qui confère au discours un caractère "polyphonique».

Les partis d'opposition veulent se livrer à des jeux partisans, a lancé le leader du gouvernement en Chambre, Tony Valeri. Le gouvernement a besoin d'avoir la confiance de la Chambre (pour continuer son travail). (Nathaëlle Morrisette, La Presse, 22 juin 2005)

Les noms de lieux, les nombres et les indications de temps sont placés plus souvent entre parenthèses qu'entre tirets. Précisons que les sigles entre parenthèses suivant une dénomination n'ont pas été retenus en tant que manifestation de la subjectivité, car ils ne font que respecter des conventions d'écriture.

Le contenu des parenthèses est beaucoup moins dépendant de la phrase hôte que les éléments placés entre tirets. Les parenthèses peuvent servir à encadrer des segments qui font un retour sur des éléments de la phrase hôte, 
mais qui ne dépendent pas syntaxiquement de celle-ci. Elles peuvent même à l'occasion renfermer des phrases complètes (comprenant une majuscule et un point).

Dans plusieurs campements, le même inspecteur constatait que l'eau était polluée par le puisard et qu'elle ne pouvait pas être potable. (Dans la même veine, le responsable de l'environnement à la Baie James M. Béland, a déclaré au Devoir qu'on avait parfois trouvé jusqu'à 130 coliformes par échantillon à certains campements [la norme est de quatre coliformes.]) (Anonyme, Le Devoir, 9 août 1975)

Les parenthèses contiennent souvent des informations accessoires, qui sont formulées plus brièvement du fait qu'elles n'ont pas à être intégrées à la structure de la phrase. Cependant, on y retrouve aussi, mais en nombre moins important, des commentaires d'une certaine longueur.

En définitive, les segments entre tirets ou parenthèses présentent des précisions ou des commentaires de même nature, si l'on fait abstraction de l'information paratextuelle figurant uniquement dans les secondes, mais ils diffèrent surtout par l'importance accordée à leur contenu, les parenthèses renfermant un discours plus en retrait, les tirets mettant en relief un propos jugé plus essentiel.

Aux fins de cette étude, nous n'avons considéré comme marques de subjectivité que les parenthèses renfermant des commentaires. La figure suivante indique l'évolution de l'usage de telles parenthèses, qu'on peut généralement gloser par «soit dit en passant ». Rappelons que tous les taux d'occurrence ont été calculés par dix mille mots.

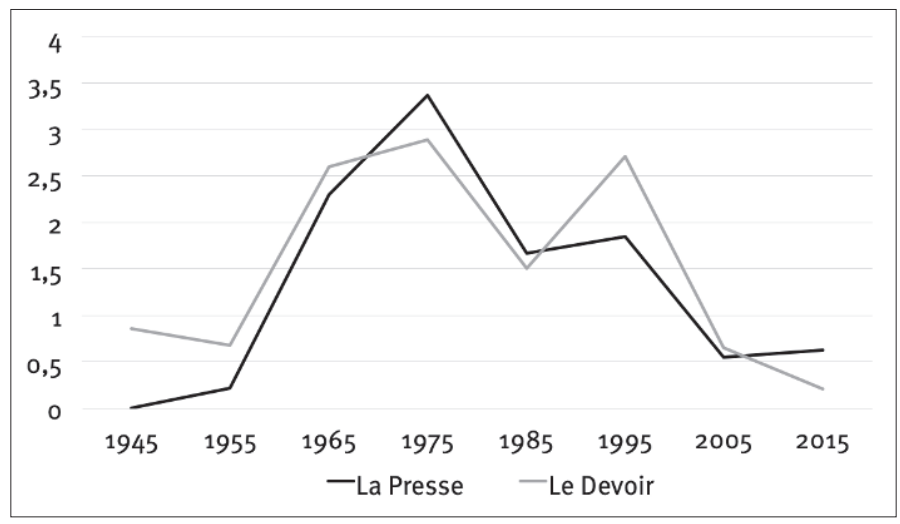

Figure 3. Taux d'occurrence par 10000 mots des parenthèses de 1945 à 2015

On constate une courbe en cloche qui présente une montée assez abrupte en 1965 et un apogée en 1975, et ce pour les deux journaux. Une baisse est enregistrée en 1985, suivie d'une remontée en 1995, puis d'une baisse considérable dans les deux dernières décennies. 
Au terme de cette section, on peut conclure que les parenthèses, contrairement aux tirets, ne présentent pas d'accroissement des taux d'occurrence dans les années 2015. De plus, les décennies où les taux d'occurrence ont été les plus élevés diffèrent d'un ponctème à l'autre. Nous développerons davantage nos conclusions à ce sujet dans la section réservée à la discussion des résultats.

\section{Mise à distance}

Les guillemets permettent, à l'instar des tirets et des parenthèses, le dédoublement de la voix énonciative parce qu'ils signalent des mots associés à un autre auteur que le journaliste a intégrés dans son texte. Le discours direct rapporté figure obligatoirement entre guillemets. Dans la presse écrite, il est fréquent que le journaliste résume les paroles d'un politicien (citation d'idées), mais en parsemant ce résumé de mots ou de groupes de mots cités textuellement qu'il encadre de guillemets ou qu'il met en italique. L'encadrement d'un mot ou d'un segment par des guillemets constitue une opération métalinguistique qui représente une «suspension de prise en charge [...] [qui] détermine une sorte de creux à combler par une autre interprétation, un "appel de glose" si l'on veut, qui s'explicite parfois, qui demeure le plus souvent implicite » (Authier, 1981, p. 128). On peut traduire un tel recours aux guillemets par «Je dis ce mot, mais pas comme je dis les autres, parce que je le dis sur le mode du comme $x$-dit» (loc. cit.), ce qui revient à dire simplement qu'il emploie dans son texte un mot qui provient d'un locuteur autre que lui. L'utilisation des guillemets de distanciation a pour effet de rendre plus vivante pour le lecteur la présentation des événements rapportés par le journaliste et laisse percevoir, dans certains cas, l'attitude ou le point de vue de ce dernier par rapport à certains mots prononcés par la personne qu'il cite. Les guillemets de distanciation mettraient en évidence des mots qui simuleraient la présence de leur auteur, procédé de concrétisation se définissant comme « une technique d'accentuation qui produit un effet de réel autour d'éléments privilégiés du développement argumentatif» (Martel, 1998, p. 119) : « [e]n reprenant les paroles qu'un individu a effectivement prononcées lors d'un événement antérieur, le discours rapporté crée l'illusion de leur présence concrète» (ibid., p.122). J. Charron explicite en ces termes le recours aux guillemets de distanciation :

Les guillemets de distanciation, en tant que discours sur le discours, fonctionnent aussi comme un procédé dialogique qui introduit dans le discours de presse une dimension d'interactivité caractéristique du journalisme contemporain. Ils agissent en effet comme un appel au lecteur en ce qu'ils introduisent dans le discours de presse une communication parallèle, comme en aparté, entre le journaliste et le lecteur, à propos d'un tiers. [...] En somme, par l'usage des guillemets de distanciation, le journaliste marque, d'un côté, une distance par rapport aux acteurs qu'il cite et, de l'autre, un rapprochement avec le lecteur. (Charron, 2006b, p.160) 
Les guillemets sont employés pour signaler l'imprécision ou l'impropriété d'un terme, un changement de registre de langue, la présence d'un anglicisme ou d'un emprunt, etc. Les effets créés par les termes guillemetés sont multiples et doivent être interprétés en tenant compte du contexte d'énonciation.

En journalisme, l'intégration de segments entre guillemets de mots ou d'expressions attribués explicitement ou implicitement à des figures politiques peut revêtir diverses valeurs énonciatives, comme l'illustre l'exemple suivant:

Branle-bas de combat hier dans le "corridor créditiste» du parlement? Et pourquoi, me direz-vous? Pour trouver de quelle juridiction fédérale ou provinciale relèvent le soleil, l'air, la pluie, la chaleur. Vous le devinez, il s'agissait encore des «machines à pluie». Ces messieurs créditistes revenaient d’une réunion avec les ministres fédéraux de la Santé et des Transports, Mlle Judy Lamarsh et M. Jack Pickersgill. Armés d'une imposante «pile» de paperasses, trois députés créditistes ont finalement réussi hier après-midi à impressionner les deux ministres. (Pierre O’Neill, La Presse, $1^{\text {er }}$ juin 1965)

Dans l'exemple ci-dessus, on note le recours abondant aux guillemets. L'auteur les utilise pour encadrer le syntagme «corridor créditiste», vraisemblablement parce que cette désignation de l'endroit du Parlement où les créditistes 4 se réunissent est plus familière qu'une indication officielle du lieu (aile, bureau) et peut-être aussi parce que c'est cette dénomination qu'emploient les habitués du Parlement. Il donne aussi aux guillemets encadrant le mot «pile» une fonction expressive, ceux-ci servent à insister sur le fait que la quantité de paperasses est imposante. Quant aux guillemets encadrant l'expression plutôt familière de «machines à pluie », ils soulignent le caractère un peu mystérieux, à l'époque, des dispositifs servant à ensemencer les nuages pour produire de la pluie.

Dans l'énoncé suivant, la journaliste souligne le terme de registre familier va-vite employé par le chef de l'opposition, terme qu'elle a retenu pour sa force expressive et peut-être, aussi, parce qu'elle est d'accord avec la critique ainsi formulée.

Dénonçant la consultation à la «va-vite» organisée par le gouvernement, qui ne reçoit en commission parlementaire que des groupes invités, André Boisclair demande «à la ministre de reporter l'adoption du projet de loi à la prochaine session parlementaire, le temps que tous ceux et celles qui le souhaitent puissent se faire entendre ». (Clairandrée Cauchy, Le Devoir, 23 novembre 2005)

Les guillemets peuvent aussi mettre en évidence de nouvelles créations lexicales qui tendent parfois à la moquerie :

4. On désigne ainsi les élus du mouvement créditiste, d’après la théorie du «crédit social ». Développée par Clifford H. Douglas, cette dernière préconise que la création monétaire exigée par l'augmentation de la production passe par la distribution d'une somme égale à chacun des citoyens. 
Une trentaine de marxistes-léninistes, réunis en fin de semaine à Montréal, ont participé à la fondation officielle de ce nouveau mouvement politique québécois. La deuxième tranche de ce congrès se tiendra le 15 mars alors que les «pétéquistes» se donneront un programme politique. (Pierre O’Neill, Le Devoir, 18 février 1975)

Dans l'extrait ci-dessus, les guillemets encadrent le nom donné aux membres du Parti des travailleurs du Québec (PTQ), formé à l'instar du mot péquistes (nom donné aux membres du $\mathrm{PQ}$, Parti québécois) à partir du sigle du parti.

La majorité des guillemets relevés dans le corpus renferment des mots ou groupes de mots qui rapportent directement des propos de politiciens et qui confèrent ainsi à l'énoncé un caractère objectif. Cependant, sur le plan de l'énonciation, non seulement le tri qui est fait parmi l'ensemble des propos tenus est le résultat d'un choix subjectif, mais les motifs de ce choix ne relèvent pas toujours d'un souci d'objectivité. Comme l'explique Patrick Charaudeau,

[l]e positionnement du sujet énonciateur [...] n'est pas toujours manifesté de façon explicite, et peut même jouer sur des apparences trompeuses en ayant l'air de s'effacer dans l'instant même où il impose son point de vue en assignant certaines places à son destinataire. (Charaudeau, 2006, § 41)

Plus simplement, les guillemets de distanciation permettent de produire divers effets de sens parfois révélateurs du point de vue du journaliste ou de l'évaluation qu'il fait d'une situation.

La figure suivante illustre une fréquence résolument croissante de l'usage des guillemets de distanciation par les journalistes.

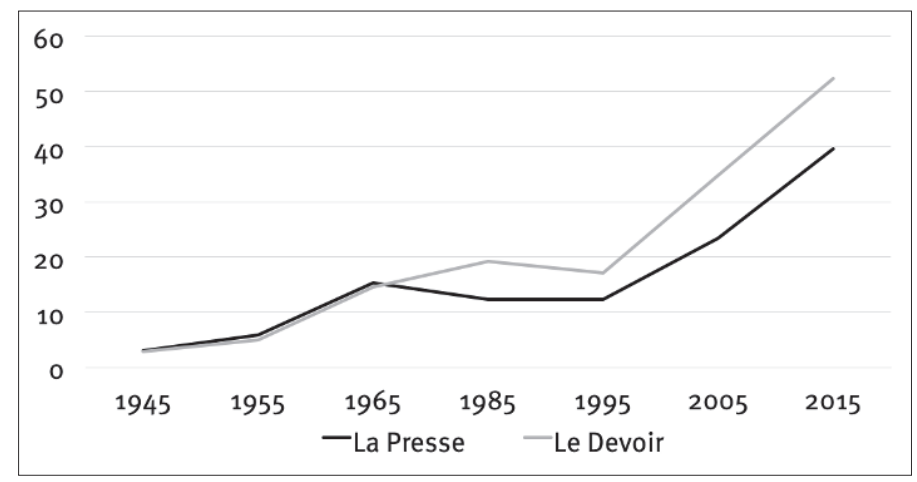

Figure 4. Taux d'occurrence par 10000 mots des guillemets de distanciation de 1945 à 2015

Les guillemets de distanciation présentent des courbes d'évolution assez similaires dans les deux quotidiens : un accroissement des occurrences 
important vers 1965 et 1975, suivi d'une légère baisse en 1985 et 1995 avant une remontée en flèche en 2005 et 2015. Les courbes témoignent de l'ampleur considérable que l'emploi de cet indicateur de subjectivité a prise au cours des décennies. On y discerne un élément de création pour agir sur le lecteur.

\section{Discussion des résultats}

Dans la présentation qu'il fait des divers paramètres de l'évolution du journalisme d’information, J. Charron précise que celui-ci,

en tant que pratique institutionnalisée de parole publique, ne peut exister et se réaliser que si les règles discursives qui le régissent sont congruentes avec celles qui régissent le fonctionnement technique, économique, organisationnel, politique, etc., des médias et du système médiatique. (Charron, 2016, p.157)

Cette congruence des paramètres nous autorise à mettre en relation la hausse des taux d'occurrence, vers les années 1965 et 1975, des ponctèmes (points de suspension, parenthèses, tirets et guillemets de distanciation) avec une conjoncture socioéconomique, politique et culturelle particulière au Québec. Les années 1960 et 1970, marquées par la Révolution tranquille5 et les revendications syndicales, ont été une période de remises en question tant sur le plan linguistique que politique et culturel. Ce climat, propice à l'expression de divers points de vue et idéologies s'opposant aux valeurs traditionnelles, a favorisé l'émergence et le développement d'une presse alternative vouée à faire valoir divers courants de pensée, ce qui a pu influer sur le style de la presse en général. J. Charron explique le changement journalistique propre à cette période en ces termes :

La période d'effervescence politique, sociale et culturelle que traverse le monde occidental dans les années 1960 et 1970 et qui est aussi une période de crise des rapports d'autorité, va donner lieu à l'établissement de relations plus conflictuelles entre les journalistes et les acteurs politiques. [...] Les journalistes vont progressivement adopter une posture plus autonome et plus critique considérant que leur rôle ne se limite pas à rapporter les faits et gestes des décideurs politiques, mais aussi à les sommer de rendre des comptes au public. (Charron, 2006b, p. 177)

Quant à Armande Saint-Jean, elle qualifie les années 1960 à 1970 de période d'éveil, de croissance, et considère les années 1970 à 1980 comme une époque de militantisme (Saint-Jean, 2002, p.139-155). Plusieurs changements politiques et sociaux marquent ces deux décennies : Révolution tranquille et oppo-

5. On désigne ainsi la période de réformes engagées par le Parti libéral du Québec à son accession au pouvoir, dans les années 1960, qui tendent notamment à la mise en place de l’État-providence. 
sition au pouvoir, influence de la télévision, début de la concentration de la presse, donc uniformisation des styles journalistiques, fondation de la Fédération professionnelle des journalistes du Québec, grèves à La Presse et au Devoir, apparition d'une presse alternative, etc.

Nos résultats corroborent la thèse selon laquelle les journalistes, durant ces années de bouleversement, ont recentré leur discours sur un rapport plus étroit avec le lecteur et ont manifesté une tendance plus marquée à influer sur son point de vue.

On note en outre une importante progression, au cours des deux dernières décennies (2005-2015), de l'utilisation des tirets et des guillemets de distanciation (en tant que marque délibérée du sentiment ou de l'attitude du journaliste par rapport à la vérité ou à la sincérité des propos qu'il rapporte). On peut conjecturer que, pour contourner l'impératif d'objectivité dans le traitement de la nouvelle, les journalistes désireux de signaler des éléments de réflexion ou de laisser percer leur point de vue ont été amenés à recourir à des moyens moins directs que ceux généralement utilisés dans l'information commentée, notamment l'emploi des tirets et des guillemets de distanciation. Quant au recul des points de suspension, les observations de J. Charron et Loïc Jacob à leur sujet fournissent une piste d'explication :

La suspension est une invitation à compléter l'énoncé, le récepteur étant censé comprendre pourquoi il n'est pas utile, nécessaire ou convenable que le locuteur complète sa pensée. L'invitation adressée au récepteur par le locuteur, bien que subtile, n'en est pas moins directe et explicite. Elle fait appel directement à la "compétence» du lecteur et à sa connivence : elle signifie, en clair, que «entre vous et moi, cela va sans dire». Le recours aux points de suspension est totalement incompatible avec un énoncé objectif qui prétendrait donner priorité à l'objet; au contraire, il accorde une telle primauté aux sujets (le locuteur et le récepteur) que la proposition sur l'état de chose n'a même pas à être explicitée, et qu'il n'est pas non plus nécessaire de préciser pourquoi. (Charron, Jacob, 1999, p.58-59; l'italique est des auteurs)

Les points de suspension, qui semblent pourtant tout à fait aptes à signaler la position du journaliste, traduisent en effet un engagement trop ostensible de celui-ci pour être vraiment compatibles avec l'esprit du journalisme d'information.

La forme en cloche de la courbe rendant compte de l'évolution du recours aux points de suspension et aux parenthèses exprimant un « décrochement énonciatif » ne conforte l'hypothèse qu'en partie. En ce qui concerne les points de suspension, les fréquences sont si faibles qu'on pourrait croire leur emploi purement fortuit. Les parenthèses, en revanche, présentent une distribution plus significative des taux d'occurrence selon les décennies. Comment expliquer qu'après une croissance assez marquée dans les années 1960 et 1970, on observe une chute importante des taux d'occurrence des parenthèses 
contenant des commentaires alors que la fréquence des tirets, qui ont essentiellement la même fonction que les parenthèses, a connu une courbe ascendante? Nos données ne fournissent pas de réponse à cette question, mais on peut formuler une hypothèse. Les parenthèses, dans la mesure où elles se distinguent des tirets par l'effet de rupture énonciative qu'elles produisent, et dans la mesure où les cas que nous avons comptabilisés sont ceux qui comportent des commentaires, c'est-à-dire des segments en général assez longs, constituent un procédé d'écriture qui tend à allonger la phrase et à alourdir sensiblement le style. Dans les années 1960 et 1970, les journalistes politiques qui écrivaient dans les quotidiens suivaient une tradition du journalisme parlementaire qui autorisait des phrases longues dans des textes prolixes et même parfois arides. À partir des années 1980, plusieurs facteurs, notamment la concurrence des nouvelles télévisées, ont pu contribuer à l'établissement de normes d'écriture axées sur l'efficacité et la lisibilité et amener les journalistes de la presse écrite à développer un style d'écriture qui soit plus vivant, plus rythmé et qui minimise la présence de phrases longues et de ruptures énonciatives comme celles auxquelles donnent lieu les parenthèses.

Quoi qu'il en soit, force est de constater que la fréquence d'emploi des différents indicateurs de subjectivité étudiés ici, bien qu'elle semble avoir fluctué de façon assez significative au fil des décennies, ne l'a pas toujours fait de façon synchronisée et selon une tendance régulière.

Les taux d'occurrence des signes de ponctuation recensés dans le corpus de nouvelles, surtout les guillemets et les tirets, confirment un changement important des pratiques du journalisme dans les années 1965-1975, période pendant laquelle le journaliste tendait à marquer de façon plus explicite sa relation avec le lecteur, à lui manifester sa confiance dans sa capacité d'interpréter ce qu'il énonçait ou sous-entendait, et à essayer par différents moyens de l'amener à partager son point de vue.

Par ailleurs, nous notons que les guillemets de distanciation et les tirets ont vu leur emploi s'accroître sensiblement dans les nouvelles au cours des deux dernières décennies. L'augmentation des taux d'occurrence de ces signes, à l'instar de celle des autres marques de subjectivité étudiées ailleurs, tend à démontrer que le journaliste accorde de plus en plus d'importance à sa relation avec le lecteur et que, par conséquent, le journalisme d'information centré sur les faits se transforme peu à peu en un journalisme de communication qui est marqué par la présence plus manifeste de l'énonciateur.

\section{Références}

Amossy Ruth, 2010, La présentation de soi : ethos et identité verbale, Paris, PUF. AUTHIER Jacqueline, 1981, «Paroles tenues à distance», dans Matérialités discursives : 
colloque des 24, 25, 26 avril 1980, Paris X-Nanterre, B. Conein, J.-J. Courtine, F. Gadet, J.-M. Marandin et M. Pêcheux éd., Lille, Presses universitaires de Lille, p.127-142.

Brin Colette, Charron Jean, Bonville Jean de éd., 2004, Nature et transformation du journalisme : théories et recherches empiriques, Sainte-Foy (Québec), Presses de l'Université Laval.

Charaudeau Patrick, 2006, «Discours journalistique et positionnements énonciatifs : frontières et dérives ", Semen, nº 22, http://journals.openedition.org/semen/2793 (consulté le 20 février 2018).

ChARRON Jean, 2016, «Du journalisme d'information au journalisme de communication », dans La parole publique, G. Martel et R. de la Garde éd., Québec, Presses de l'Université Laval, p.151-168.

- 2006a, "Subjectivation du discours du journalisme politique au Québec, 19451995 ", Mots. Les langages du politique, n 82, p. 81-94.

— 2006b, «Journalisme, politique et discours rapporté : évolution des modalités de la citation dans la presse écrite au Québec, 1945-1995 ", Politique et Sociétés, vol. XXV, n०2-3, p. 147-181.

CHARRON Jean, JACOB Loïc, 1999, «Énonciation journalistique et subjectivité : les marques du changement», Études de communication publique, nº 14, p.1-68.

DALHET Véronique, 2003, Ponctuation et énonciation, Matoury (Guyane), Ibis rouge éditions.

De Beaugrande Robert, 1984, Text Production: Toward a Science of Composition, Norwood, Ablex Publishing Corporation.

FORGET Danielle, 2000, "Les insertions parenthétiques », Revue québécoise de linguistique, vol. XXVIII, $\mathrm{n}^{\circ}$ 2, p. 15-28.

FrançoIs Guillaume, 2011, «Étude comparée du fonctionnement des parenthèses et des tirets ", Discours, nº 9, https://journals.openedition.org/discours/8542 (consulté le $1^{\text {er }}$ octobre 2018).

MaIn GUENEAU Dominique, 1976, Initiation aux méthodes de l'analyse du discours : problèmes et perspectives, Paris, Hachette.

MARTel Guylaine, 1998, Pour une rhétorique du quotidien, Québec, Ciral.

PADIOLEAU Jean-Gustave, 1976, «Systèmes d'interaction et rhétoriques journalistiques », Sociologie du travail, $18^{\mathrm{e}}$ année, $\mathrm{n}^{\circ} 3$, p. 256-282.

PétIlLon-Boucheron Sabine, 2002, Les détours de la langue : étude sur la parenthèse et le tiret double, Louvain, Paris, Dudley (Mass.), Peeters.

Schudson Michael, 1996, The Power of News, Cambridge, Harvard University Press.

SAINT-JEAN Armande, 2002, Éthique de l'information : fondements et pratiques au Québec depuis 1960, Montréal, Presses de l'université de Montréal.

SERÇA Isabelle, 2010, Les coutures apparentes de la Recherche : Proust et la ponctuation, Paris, Honoré Champion. 


\section{Résumé / Abstract / Compendio}

\section{Sur quelques marques de subjectivité dans le journalisme d'information politique de 1945 à 2015 au Québec}

Partant du constat que le journalisme d'information politique a subi des transformations et qu'il affiche un discours plus empreint de subjectivité, cette recherche empirique porte sur l'analyse et l'évolution du recours à quatre variables linguistiques : les points de suspension, les tirets, les parenthèses et les guillemets de distanciation. Ces ponctèmes traduisent à la fois un engagement du journaliste en tant qu'énonciateur et un rapprochement de celui-ci avec son lecteur.

Mots-clés : journalisme, subjectivité, énonciation, ponctuation, engagement

\section{On some subjectivity marks in political information journalism from 1945 to 2015 in Quebec}

Based on findings which indicate that political information journalism has undergone transformations which evidence a more subjective discourse, this empirical research focuses on the analysis and evolution of the use of four linguistic variables: ellipsis, dashes, parentheses, and distancing quotation marks. These punctuation marks reflect both the presence of the journalist as the enunciator and a proximity to his/her reader.

Keywords: journalism, subjectivity, enunciation, punctuation, involvement

\section{Algunas marcas de subjetividad en el periodismo de información política de 1945 a 2015 en Quebec}

Basado en la observación de que el periodismo de información politíca ha sufrido transformaciones que de muestran un discurso más subjetivo, esta investigación empírica se centra en el análisis y la evolución del uso de cuatro variables lingüísticas: los puntos de suspensión, los guiones, los paréntesis y las comillas. Estas puntuaciones reflejan tanto el compromiso del periodista como informante como la proximidad a su lector.

Palabras claves: periodismo, subjetividad, enunciación, puntuación, compromiso 